\title{
Actuator Linearisation for Multivariable Control Applications
}

\author{
John Ringwood \\ Dept. of Electronic Engineering \\ National University of Ireland, Maynooth \\ Maynooth, Co. Kildare, Ireland \\ Email: john.ringwood@eeng.nuim.ie
}

\author{
Annraoi dePaor \\ Dept. of Electrical and Electronic Engineering \\ National University of Ireland, Dublin \\ Belfield, Dublin 4, Ireland \\ Email: annraoi.depaor@ucd.ie
}

\begin{abstract}
Fixed speed actuators have a characteristic nonlinear transient response. While this may not present a problem for single-input, single-output (SISO) control systems containing such actuators, the mixture of different fixed speed actuators is usually problematic in multivariable control applications. This paper presents two methods which allow such nonlinear actuators to present a linear response and therefore allow their incorporation in linear multivariable control design methods. The first method is based on a closed-loop describing function approximation, while the second uses an equivalent nonlinearity concept to linearise the relay which represents the fixed-speed limitation. A simulation study, demonstrating the performance of both schemes in a multivariable control setting, is presented.
\end{abstract}

Index Terms-Actuator, linearisation, describing function, equivalent nonlinearity, multivariable control

\section{INTRODUCTION}

There is a wealth of literature on actuator linearization. Many studies use feedback linearisation, which uses an inverse actuator model and relies on the sensitivity reduction of the feedback to compensate for any residual nonlinearity, e.g. [1]. However, this cannot be applied to relay-type systems, since the relay represents a non-invertible characteristic. A number of studies look at small signal linearised representations, e.g. [2], but these are only valid for a very small range of operation. Most studies treat saturating actuators, e.g. [3], [4], but these researchers consider an essentially linear actuator, which has an amplitude limitation. Finally, [5] considers an electro servohydraulic valve, but linearises the static characteristic. The approach in this paper is to deal with the complete actuator servo loop and to develop a compensation method, which achieves something close to exact linearisation of the servo loop, over its complete range of operation.

Nonlinear actuators of the form shown in Fig.1 are found in many systems, both hydraulic and electrical. The effect of the relay shown is often manifested as a controller output which has only 'raise' or 'lower' signals, limiting the maximum rate of change of the output, often for safety reasons or due to physical limitations. Electrical fixed-speed (ac) drives are also cheaper than their variable-speed counterparts, with speed fixed by the supply frequency.

This paper develops two compensation methods, one based on the concept of the use of a dither signal to transform the relay characteristic into an 'equivalent nonlinearity' [8] while

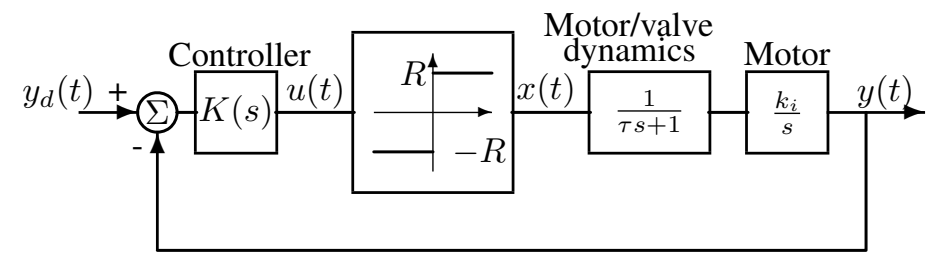

Fig. 1. Fixed-speed actuator system

the other method determines a describing function approximation [6] for the complete actuator loop. The operation of both methods is demonstrated in both single-loop and multivariable control settings and the duty cycle implied by the switching control signals is examined.

\section{EQUiVALENT NON-LINEARITY}

The concept of an equivalent non-linearity for a nonlinear characteristic subject to a (relatively) high frequency dither signal has been traced back to J.C. Lozier of Bell Labs in 1950 [7], [8]. It can be used both as an analysis technique to examine the effect of a combination of a high frequency dither signal and a non-linearity, or as a synthesis technique, where a dither signal is specially constructed and injected into a system in order to produce a more desirable (probably less severe) non-linear function. It is the latter case that is addressed in this paper.

\section{A. Equivalent non-linearity concept}

The equivalent nonlinearity concept addresses the system as shown in Fig.2. The original non-linearity is specified in $y=f(u)$, while the equivalent non-linearity of $f()$ with the addition of the dither signal is specified by $\bar{y}=g(r)$.

It can be shown that the equivalent non-linearity of the single-valued function $x(t)=f(u(t))$, with $\mathrm{u}=\mathrm{r}+\mathrm{d}(\mathrm{t})$, where $\mathrm{r}$ is a (relatively) low frequency signal and $\mathrm{d}(\mathrm{t})$ a (relatively) high frequency dither signal, is given by $g(r)$, where:

$$
\bar{y}=g(r)=\int_{-\infty}^{\infty} f(u) p(u-r) d u
$$

and $p()$ is a weighting function related to a probability density function on $d(t)$. The technique relies on two assumptions: 


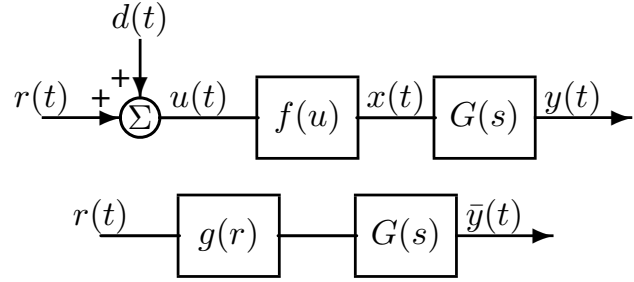

Fig. 2. Equivalent non-linearity concept

- The frequency of the dither waveform, $d(t)$, lies above the bandwidth of the dynamic system, $G(s)$, which follows the non-linear element, and

- The spectral difference between $r(t)$ and $d(t)$ is such that, over a dither period, $T$, no significant error is incurred by regarding $r(t)=r$ as constant.

\section{B. Calculation of the equivalent non-linearity}

In general, for a single-valued non-linearity, $f()$, with input $u(t)=r+d(t)$, with $d(t)$ being the dither signal [8], and $\mathrm{r}$ being relatively constant over the dither period,

$$
y=f(r+d(t))
$$

Let $p(q) d q$ be the probability that, for any time $t$, chosen at random, $d(t)$ lies in the range $q$ to $q+d q$, with $p(q)$ being the probability density function for the dither. When $d(t)$ has the value $q$,

$$
y=f(r+q)
$$

The expected value for $y$ is:

$$
\bar{y}=\int_{-\infty}^{\infty} f(r+q) p(q) d q
$$

To calculate $p(q)$, let $F(q)$ be the probability that $d(t)$ lies above $q$,

$$
F(q)=\int_{q}^{\infty} p(q) d q=\int_{\infty}^{q} p(q) d q
$$

and $p(q)$ can now be determined from:

$$
\frac{d F(q)}{d q}=-p(q)
$$

\section{A relay with triangular dither}

The equivalent nonlinearity for an ideal relay with a triangular dither signal on the input will now be evaluated. A triangular dither signal is a powerful one and has the capability significantly linearise a relay, as will be demonstrated. The triangular dither signal is shown in Fig.3 and a relay with limits of $\pm R$, as shown in Fig.1, is the non-linearity under study.

For a triangular dither signal of amplitude $D$, as shown in Fig.3, $F(q)$ may be evaluated as the fraction of time that $d(t)$ spends above $q$, and is easily seen to be:

$$
\begin{aligned}
F(q) & =A / B \text { for }-D<q<D \\
& =\frac{D-q}{2 D}
\end{aligned}
$$

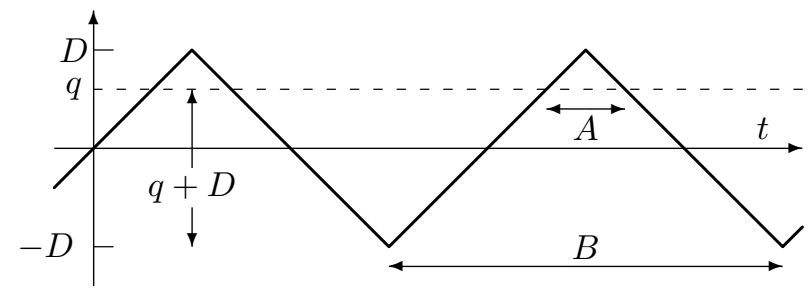

Fig. 3. Triangular dither signal

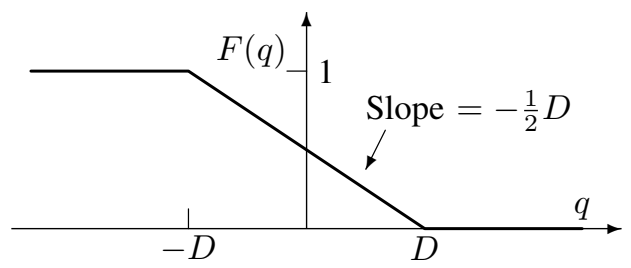

Fig. 4. Function $F(q)$ for triangular dither

Also

$$
\begin{aligned}
& F(q)=0 \text { for } q>D \\
& F(q)=1 \text { for } q<-D
\end{aligned}
$$

The form of $F(q)$, for a triangular dither signal, is shown in Fig.4 and the corresponding probability density function, $p(q)$, shown in Fig.5.

We can now evaluate the effect of the dither signal on the relay by evaluating equation (1). The integral in (1) represents the common area under the function formed by the product $f(r) p(r-u)$ or, equivalently, $p(r) f(u-r)$. This is easily calculated graphically, as shown in Fig.6. The area under the negative portion of the $p(r) f(u-r)$ curve is $\frac{R}{2 D}(D-r)$ while the area under the positive portion is $\frac{R}{2 D}(D+r)$, giving a net area of $\frac{R r}{D}$. The equivalent nonlinearity is therefore:

$$
\bar{y}=\frac{R}{D} r \text { for }-D<r<D
$$

with

$$
\begin{aligned}
& \bar{y}=D \text { for } r>D \\
& \bar{y}=-D \text { for } r<-D
\end{aligned}
$$

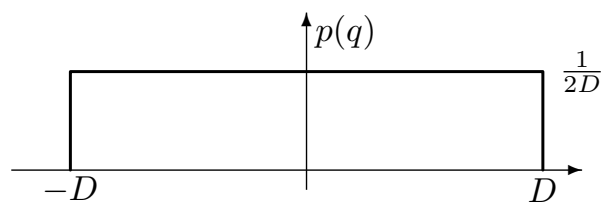

Fig. 5. Probability density function for triangular dither 


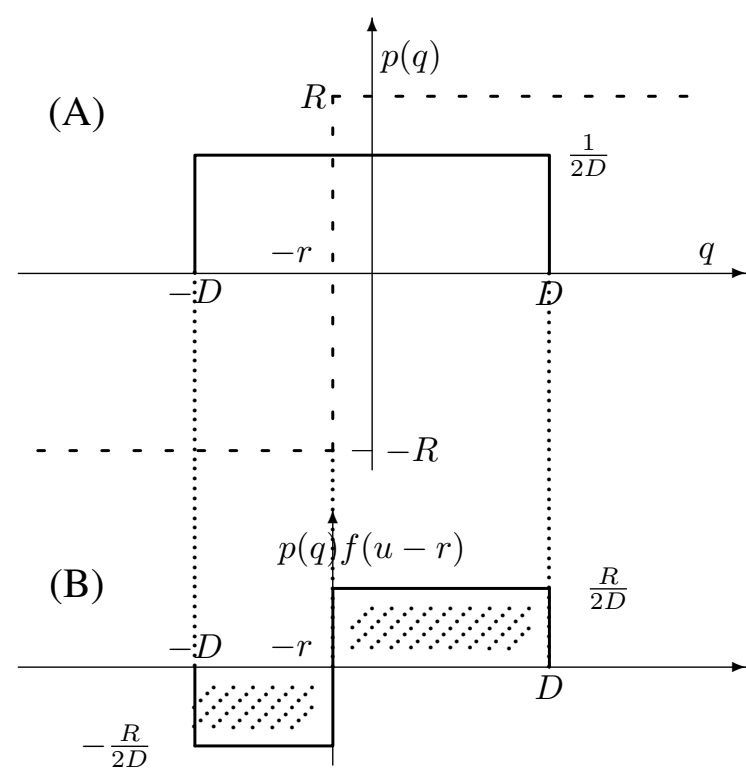

Fig. 6. Evaluation of convolution product

which is linear in $r$ between the relay saturation limits!. Providing the 'equivalent nonlinearity', $g(r)$ operates in its linear region, the closed-loop transfer function of the actuator system in Fig.1, with dither injected, is:

$$
T_{e n}=\frac{1}{1+\left(D / K(s) G_{v}(s) R k_{i}\right) s}
$$

If, for the present, we neglect the motor/valve dynamics, and we choose $K(s)=k_{o}$, we get:

$$
T_{\text {en }}=\frac{1}{1+\left(D / k_{o} k_{i} R\right) s}
$$

which is a first order transfer function with time constant

$$
\tau_{d}=\frac{D}{k_{o} k_{i} R}
$$

\section{DesCRIBING FunCTION APPROXIMATION}

The describing function (DF) gives a representation for a nonlinear characteristic in terms of a frequency and amplitudedependent gain. For static nonlinear characteristics, such as the relay, the DF has an amplitude dependence only. In the development of the describing function, a 'test' sinusoidal signal is injected into the nonlinearity and the transmission 'gain' of the nonlinearity with respect to the fundamental component of the output signal calculated. It is assumed that the harmonics on the output are attenuated by low-pass elements following the nonlinearity, which is reasonable in this case, given the presence of the integrating function of the motor and the valve/motor dynamics.

For the relay in Fig.1, we can determine the describing function straightforwardly [6] as:

$$
N_{r}(u)=\frac{4}{\pi|u|}
$$

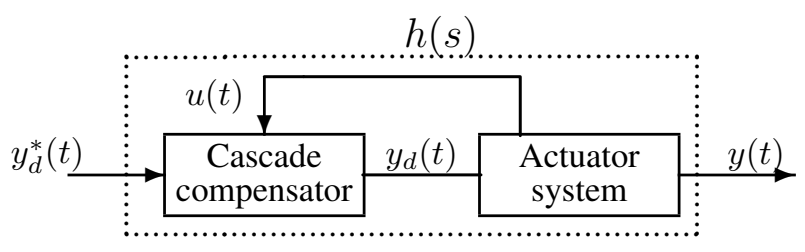

Fig. 7. Amplitude dependent cascade compensator

where $u$ is the amplitude of the signal entering the relay. If, for the present, we neglect the motor/valve dynamics and, initially (at least) let $K(s)=k_{o}$, evaluating the closed-loop differential equation for the actuator system of Fig.1, using the DF approximation of Eq.(17) for the relay, yields:

$$
\pi|u(t)| \dot{y}(t)-4 k_{o} k_{i}\left(y_{d}(t)-y(t)\right)=0
$$

and the closed-loop actuator system approximates a linear, unity-gain, first-order dynamical system, with an amplitude dependent gain, with time constant:

$$
\tau_{e}=\frac{\pi|u|}{4 k_{o} k_{i}}
$$

If an amplitude dependent compensator, defined by:

$$
\tau_{d} \dot{y}_{d}(t)+y_{d}(t)=\tau_{e}(u(t)) \dot{y}_{d}^{*}(t)+y_{d}^{*}(t)
$$

is now placed in cascade with the actuator system of Fig.1, as shown in Fig.7, the overall system transfer function is effectively:

$$
h(s)=\frac{1}{1+\tau_{d} s}
$$

where $\tau_{e}$ is a design parameter and chosen subject to actuator speed limitations (see Section IV-A). It may be noted that the cascade compensator in (20) may easily be implemented using the state-space form:

$$
\begin{aligned}
\dot{x}(t) & =A x(t)+B(t) y_{d}^{*}(t) \\
y_{d}(t) & =C x(t)+D(t) y_{d}^{*}(t)
\end{aligned}
$$

with $A=-\left(1 / \tau_{d}\right), \mathrm{C}=1$ and the dependence on $|u|$ confined to $B(t)=\left(\tau_{d}-\tau_{e}(u)\right) / \tau_{d}^{2}$ and $D(t)=\tau_{e}(u) / \tau_{d}$.

\section{Actuator Controller Calculations}

\section{A. Choice of $\tau_{d}$}

Both the equivalent nonlinearity (EN) and describing function (DF) approaches have, to a first approximation, the capability to reduce the actuator system in Fig. 1 to a linear, first order transfer function. In the EN approach, the addition of a dither signal is required, while the DF approach requires the use of an amplitude-dependent cascade compensator.

Now, we will address the choice of $\tau_{d}$. While significant freedom in the choice of $\tau_{d}$ is available, it is important that we establish a lower bound on $\tau_{d}$ so that we don't try to violate the rate limit of the actuator output, which is given by $k_{i}$. Otherwise, nonlinear behaviour will result. 
Let us consider a step response, and try to determine the minimum $\tau_{d}$ which will retain linear behaviour. The response of the system described by (21) is:

$$
y_{\text {step }}=A_{s}\left(1-e^{-\frac{t}{\tau_{d}}}\right)
$$

where $A_{s}$ is the magnitude of the step function. The maximum slope of the step response is determined by:

$$
\left.\frac{d y_{\text {step }}}{d t}\right|_{t=0}=\left.\left(A_{s} / \tau_{d}\right) e^{-\frac{t}{\tau_{d}}}\right|_{t=0}=A_{s} / \tau_{d}
$$

To avoid violation of the maximum slope, $k_{i}$, we must have:

$$
A_{s} / \tau_{d}<k_{i}
$$

or

$$
\tau_{d}>A_{s} / k_{i}
$$

In order to get the fastest linear response, set $\tau_{d}=A_{s} / k_{i}$. We now determine the actuator controllers required to obtain this limiting condition.

\section{B. Controller calculations}

Again neglecting the motor/valve dynamics initially, we have from (16) that:

$$
\tau_{d}=\frac{D}{k_{o} k_{i} R}=A_{s} / k_{i}
$$

giving:

$$
k_{o}=\frac{D}{A_{s} R}
$$

for the maximum rate linear response, using the EN framework. In the DF framework, we simply choose $\tau_{d}$ (in Eq.(21)) from Eq.(27), with the choice of $K(s)$ being relatively unimportant. However, in the case that the relay contains some deadzone (often artificially introduced to prevent limit cycling), $K(s)$ may be chosen as a constant gain, $k_{o}$ in conjunction with the dead-zone value (note that $k_{o}$ effectively scales the dead-zone quantity).

\section{RESULTS}

\section{A. Single loop responses}

With parameters of $k_{i}=2$ and $\tau=0.01 \mathrm{~s}$, we compute the responses of two actuators to steps of amplitude $A_{s}=10.0$. One actuator is compensated using the DF technique, while the other is subject to a triangular dither signal of amplitude 2 . The relay is symmetrical and saturates at \pm 1 . Fig. 8 shows the responses of both systems, together with a linear system with time constant $\tau_{d}=A_{s} / k_{i}=5 \mathrm{~s}$. From (29), $k_{o}=0.2$. Fig. 8 also shows the response for an uncompensated actuator.

Clearly, the response achieved with both linearising methodologies is very close to the linear equivalent, with just a minor mismatch in the case of the EN methodology. Though a linear actuator response is achieved, the price to be paid is an increase in the time taken to achieve the setpoint. In many applications, the faster response may be desirable, with little negative impact of the nonlinear transient response. This is most likely the case when the actuators are used in isolation. However, when such an actuator system is contained within a larger control

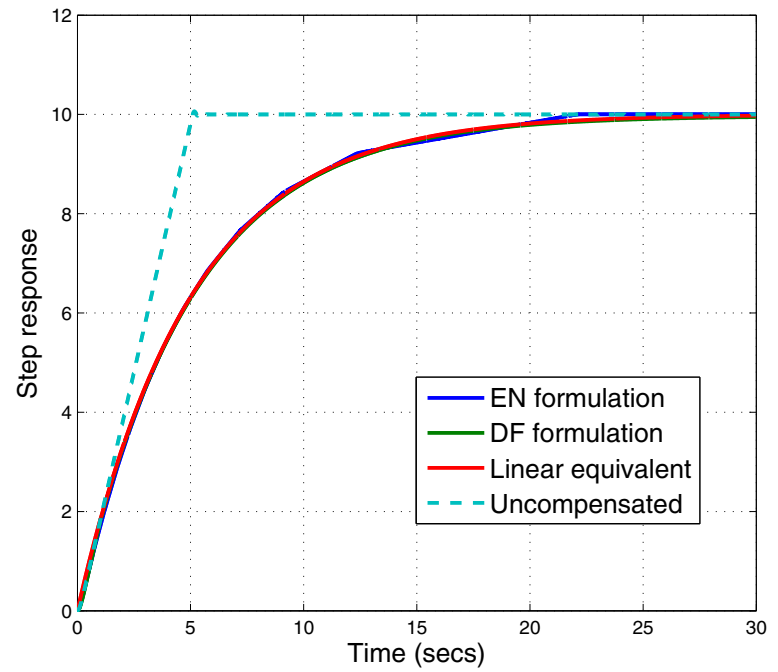

Fig. 8. Uncompensated and compensated single actuator response

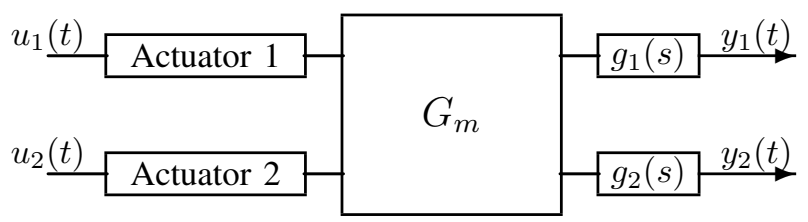

Fig. 9. Example multivariable system

strategy, particularly involving multivariable interactions, there is significantly stronger motivation to obtain a linear response, at the expense of some degradation in transient response time. The following example illustrates such a scenario.

\section{B. Multivariable control example}

In this example, we consider the case where, for simplicity, all multivariable interaction takes place in a matrix of constant gains. The system under study, shown in Fig.9 has two inputs and two outputs and both inputs actuate the system via nonlinear actuator systems of the form shown in Fig.1, but with different parameters. The dynamical blocks on the output are similar single-input, single-output (SISO) dynamics described by $g_{1}(s), g_{1}(s)$, where:

$$
G_{m}=\left(\begin{array}{ll}
2 & 1 \\
3 & 3
\end{array}\right), g_{1}(s)=g_{2}(s)=\frac{1}{5 s+1}
$$

The actuator parameters are given in Table I.

The multivariable system described above is indicative of a number of application areas, including those arising in steel

TABLE I

ACTUATOR PARAMETER VALUES

\begin{tabular}{|c|c|c|}
\hline & $k_{i}$ & $\tau$ \\
\hline Actuator 1 & 2 & 0.1 \\
Actuator 2 & 0.5 & 0.3 \\
\hline
\end{tabular}




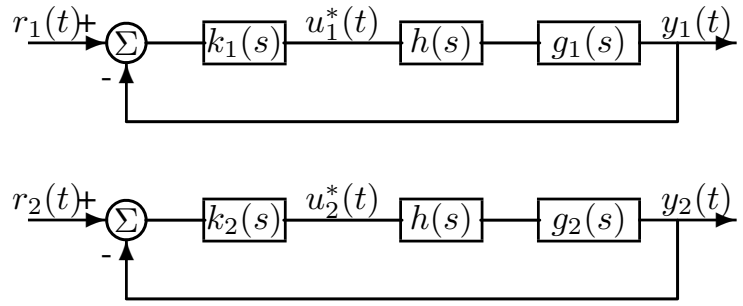

Fig. 10. Diagonalised system

rolling [9] and in boiler systems [10], where the dynamics of the multivariable interaction are significantly faster than other SISO dynamics in the system. Typically, different causal inputs to the system, with diverse inertias and ranges of movement, require different actuator systems.

A typical strategy to control the system of Fig.9 is to diagonalise the system and apply single-loop compensation. However, this can only be achieved if the actuators can be linearised. Furthermore, since neither actuator can be driven beyond its maximum rate, as detailed in (27), the fastest actuator will need to be slowed down to the minimum time constant achievable by the slowest one. We therefore choose $\tau_{d}=A_{s} / k_{i}$ for Actuator 2, where $A_{s}$ in this situation represents the maximum range of movement. For this example, we will assume that $A_{s}=5$.

So, we appropriately compensate both actuators to align them with the transfer function, $h(s)$ in (21) yielding the transfer function matrix:

$$
G_{M}(s)=\left(\begin{array}{cc}
g_{1}(s) & 0 \\
0 & g_{2}(s)
\end{array}\right) G_{m} h(s)
$$

Since $G_{m}$ is non-singular, we diagonalise the system using

$$
G_{m}^{-1}=\left(\begin{array}{cc}
1 & -\frac{1}{3} \\
-1 & \frac{2}{3}
\end{array}\right)
$$

and apply single loop compensation to the remaining two independent dynamical SISO systems, in the configuration:

$k_{1}(s)$ and $k_{2}(s)$ can be chosen to equalise the dynamics in each path, if desired, or individual (decoupled) responses can be achieved. Let us choose:

$$
k_{1}(s)=k_{p 1}+\frac{k_{i 1}}{s}=k_{2}(s)
$$

with $k_{p 1}=0.8$ and $k_{i 1}=0.1$.We have, from (27) that $\tau_{d}^{\min }=$ 10. This gives:

$$
k_{o}^{1}=0.1, k_{o}^{2}=0.4
$$

The responses, with uncompensated actuators, to steps (of amplitude 5 units) in inputs 1 and 2 respectively, are shown in Fig.11, while the responses using compensated actuators is shown in Fig.12.

Though the responses of the system with uncompensated actuators is slightly faster, there are some undesirable interactions between channels which cannot be eliminated. Good channel separation is seen to be achieved for the system with (a) Step in Input 1

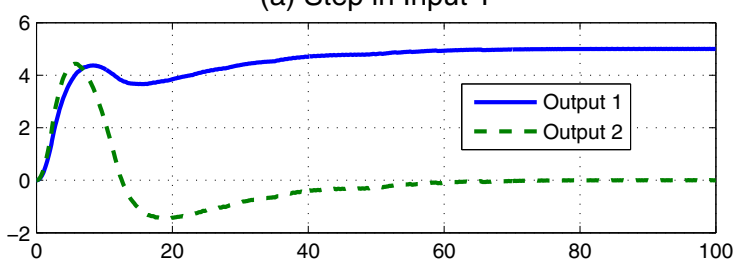

(b) Step in Input 2

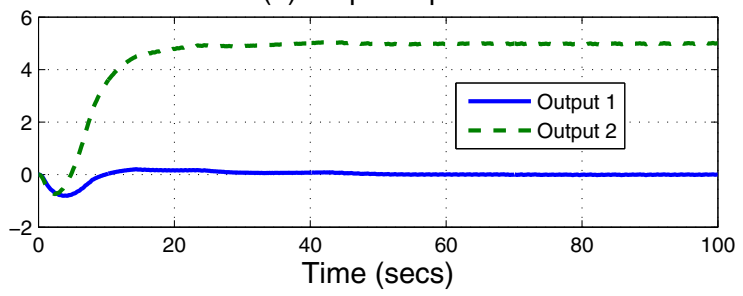

Fig. 11. Multivariable system response (uncompensated)

(a) Step in Input 1

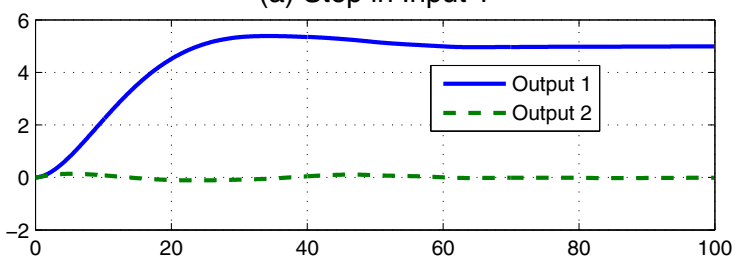

(b) Step in Input 2

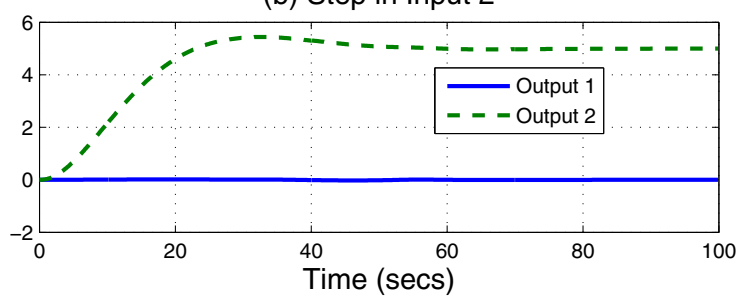

Fig. 12. Multivariable system response (compensated)

actuator compensation. In Fig.12, results are shown for the EN formulation, with the DF formulation yielding comparable results.

\section{DISCUSSION}

There are a number of issues, arising in relation to the actuator linearisation methods presented, which deserve some comment. It should be noted that both linearisation methods presented in this paper effectively perform some form of switching action across the relay. This is an effective method of propagating an 'analogue' signal across the relay, where we rely on the low-pass components following the relay to perform an integrating, or averaging, operation. Alternative switching methodologies have been proposed elsewhere, for example in [11], which employs the use of pulse-width modulation (PWM) to assist in linearising the actuator over time. In [11], the width of the pulses is proportional to the magnitude of the relay input.

Switching, in some systems, may cause unacceptable wear 
on components, for example in hydraulic systems, where a switching action would require rapid changes in the direction of a mechanical spool valve. Therefore, the techniques presented here are limited to electrical actuator systems. Note that the degree of switching can be loosely controlled by the introduction of dead-zone into the relay characteristic, at the possible expense of some small steady-state error in the actuator output. This is particularly true of the DF formulation, since the amount of switching depends on the distance of the actuator output from the setpoint. However, this is less so in the case of the EN, where a consistent dither signal is applied. Nevertheless, some experimentation with the dither amplitude, or possible modulation of the dither amplitude with actuator error, may lead to a more acceptable degree of switching.

It should also be noted that the effect of both dead-zone in the relay and any backlash on the output may be taken into account in both the DF and EN formulations, so compensation for these effects is also possible.

In the calculations of Sections III and IV, we neglected the unity-gain valve/motor dynamics. For the range of dynamical time constants considered, these dynamics were not seen to significantly affect the results. However, if desired, a more accurate calculation of the cascade compensator in Fig.7 (as opposed to the simple form in Eq.(20)) and $K(s)$ (as opposed to the simple form in Eq.(29)) can be made, resulting in a two-degree-of-freedom controller in the case of $K(s)$ for the system with dither. Nevertheless, this significant increase in complexity is unlikely to be justified in relation to the performance improvement.

Finally, it should be borne in mind that the design of the outer multivariable controller is not entirely independent of the actuator constraints, even post-linearisation. Note that the minimum time constant calculation in Eq.(27) depends on the setpoint demands at the actuator input and violation of these design limits will result in nonlinear behaviour (i.e. the output will be rate-limited) and multivariable decoupling lost. This could easily be demonstrated by using a more aggressive set of dynamic controllers for $k_{1}(s), k_{2}(s)$. In general, increasing the outer loop proportional gain beyond a certain threshold will cause an instantaneous input to the actuator which may exceed the design specification, $A_{s}$. The use of derivative-type action is also likely to lead to significant problem in this regard. Integral action, on the other hand, generally provides a slow, incremental, actuator demand and is unlikely to violate the input design limits.

\section{CONCLUSION}

This paper presents two relatively straightforward techniques for linearising fixed-speed actuators. While the nonlinear transient response from fixed-speed actuators may not be a particular issue for stand-alone actuator systems, linearisation is required if acceptable performance of multivariable system, incorporating such actuators, is to be achieved. Simple calculations are provided to determine the maximum speed (minimum time constant) of the linearised actuator systems, leading to straightforward actuator controller calculations. Both lineari- sation schemes produce a switching effect on the relay output which can, to some extent, be mitigated by the incorporation of appropriate dead-zone in the relay, at the expense of some steady-state accuracy.

\section{REFERENCES}

[1] X. Ma and G. Tao, Adaptive actuator compensation control with feedback lineaisation, IEEE Trans. Auto. Control, Vol.45, No.9, pp 1705-1710, 2000.

[2] X. Lin and S. Scavarda, Linearized analysis of an electropneumatic servovalve/long pneumatic actuator assembly using bond graph, Proc. Int. Conf. Syst., Man. Cybern., Vol.1, pp 555-560, 1993.

[3] J. Grantz, S. Valluri and M. Soroush, Discrete-time nonlinear control of processes with actuator saturation, AIChE Journal, Vol.44, No.7, pp 17011705,1998

[4] C. Gokcek, P.T. Kabamba and S.M. Meerkov, An LQR/LQG theory for systems with saturating actuators, IEEE Trans. Auto. Control, Vol.46, No.10, pp 1529-1542, 2001.

[5] H. Hahn, A. Piepenbrink and K.D. Leimbach, Input/output linearization control of an electro servo-hydraulic actuator, Proc. $3^{\text {rd }}$ IEEE Conf. on Control Applications, Vol.2, pp 995-1000, 1994.

[6] D.P. Atherton, Nonlinear Control Engineering, Van Nostrand Reinhold, New York, 1982.

[7] J.C. Lozier, Carrier-controlled relay servos, Electrical Engineering, Vol.69, pp 1052-1056, 1950.

[8] R.J. Simpson and H. M. Power, Applications of high frequency signal injection in non-linear systems, Int. J. Control, Vol.26, No.6, 917-943, 1977.

[9] J.V. Ringwood, Shape control systems for Sendzimir steel mills, IEEE Trans. on Control Systems Technology, Vol.8, No.1, pp 70-86, 2000.

[10] B. Molloy, Modelling and predictive control of a drum-type boiler, $\mathrm{PhD}$ thesis, Dublin City University, 1997.

[11] K. Dutton, An investigation into the design and performance of a shape control system for a Sendzimir mill, Ph.D. dissertation, Sheffield City Polytechnic, 1983. 